\title{
Spectral Efficiency Improvement Techniques In Massive Mimo For 5G Communications
}

\author{
Naraiah R ${ }^{\text {a }}$, Dr. Mukesh Tiwari ${ }^{\mathrm{b}}$ and Dr.Patteti Krishna \\ a Research Scholar, Dept. of Electronics and Communication Engineering, \\ Sri Satya Sai University of Technology \& Medical Sciences, Sehore, Bhopal Indore Road, Madhya Pradesh, India \\ b Research Guide, Dept. of Electronics and Communication Engineering, \\ Sri Satya Sai University of Technology \& Medical Sciences, Sehore, Bhopal Indore Road, Madhya Pradesh, India \\ ${ }^{c}$ Research Co-Guide,Ambedkar Institute of Advanced Communication Technologies \& Research (AIACT\&R), \\ Geeta Colony, Delhi
}

Article History: Received: 11 January 2021; Accepted: 27 February 2021; Published online: 5 April 2021

\begin{abstract}
Wireless communications has gotten one of the quickest developing zones in our advanced life and makes colossal effect on practically every component of our day by day life. $5 \mathrm{G}$ should support a large number of new applications with a wide assortment of prerequisites, including higher pinnacle and client information rates, diminished dormancy, improved indoor inclusion, expanded number of gadgets, etc. The normal traffic development in at least a long time from now can be fulfilled by the consolidated utilization of more range, higher spectral efficiency, and densification of cells. The increment in spectral effectiveness will improve the throughput of the system which straightforwardly serves the Enhanced Mobile Broad band use instance of the $5 \mathrm{G}$ assistance. In massive Multiple-Input Multiple-Output (M-MIMO) systems few hundred quantities of antennas are conveyed at each base station (BS) to serve a moderately modest number of single-reception apparatus terminals with multiuser, giving higher information rate and lower idleness. Massive Multiple-Input Multiple-Output is the arising innovation in cell system for higher information rate correspondence. It utilizes enormous number of communicating reception apparatus at the base station which is made conceivable by the radio wire cluster which can be electronically steerable and adequately utilized for shaft framing. Spectral proficiency is the vital boundary to be improved in expanding throughput. The system execution under different commonsense limitations and conditions, for example, restricted soundness block length, number of base station (BS) antennas, and number of dynamic clients are assessed through simulation.
\end{abstract}

Keywords: Multiple-Input Multiple-Output (MIMO), Base Station (BS), 5G, Wireless communications, spectral efficiency.

\section{Introduction}

In a couple of years, a few investigations have been centered around massive MIMO systems which assume a significant part in arising patterns in correspondence organizations. The massive MIMO handset contains the different number of brilliant radio wire clusters, which empower to get higher spectral productivity and energetically effective to be accomplished. In different antennas were appended to an exhibit structure to sort out the base station and versatile stations of the wireless correspondence link, to deal with the sign suitable way to improve the system execution. The multi-bar framing needs to join the multiple signs got or sent by a variety of antennas. Planning and execution challenges are related with its design and applications. The massive MIMO antennas are coordinated with beamforming cluster reception apparatus innovations for cutting edge cell correspondence and conveyed in 2020 as suggested. Different quantities of correspondence model and antennas can't expand the limit of wireless correspondence systems to moderate the multipath blurring and channel obstruction. The beamforming strategy is improving sign radiation in like manner to the climate. In correspondence organizations, transmitter and recipient utilized the beamforming signal transmission from base station to versatile station with multiple antennas. The critical subject of empowering the beamforming in the correspondence organizations, to upgrade the force limit and limit the impedance.

There are a few techniques proposed to improve spectral productivity. Massive MIMO is outstanding amongst other way to deal with improve spectral proficiency and furthermore it supports with the equipment system having huge number of antennas as in a 5G system. Thus massive MIMO approach will be a superior answer for improving SE for 5G systems. Upgrading the quantity of reception apparatus is accounted for. The cell system has a few base Station which works in a lucid design. It gives better cluster acquire and spatial goal that permits keeping up vigor to entomb cell impedance. The forceful multiplexing in massive MIMO has contributed for the improvement of by and large effectiveness.

\subsection{Evolution of Cellular Networks}

The portable correspondence period began in the mid-1980s, and from that point forward, versatile correspondence has encountered gigantic development in the previous few decades. Cell networks have advanced from $1 \mathrm{G}$ to $5 \mathrm{G}$ and past. All cell networks are made out of base stations, client gear (telephones), and center organizations. The development from $1 \mathrm{G}$ to $6 \mathrm{G}$ is summed up in Figure 2.1 . 


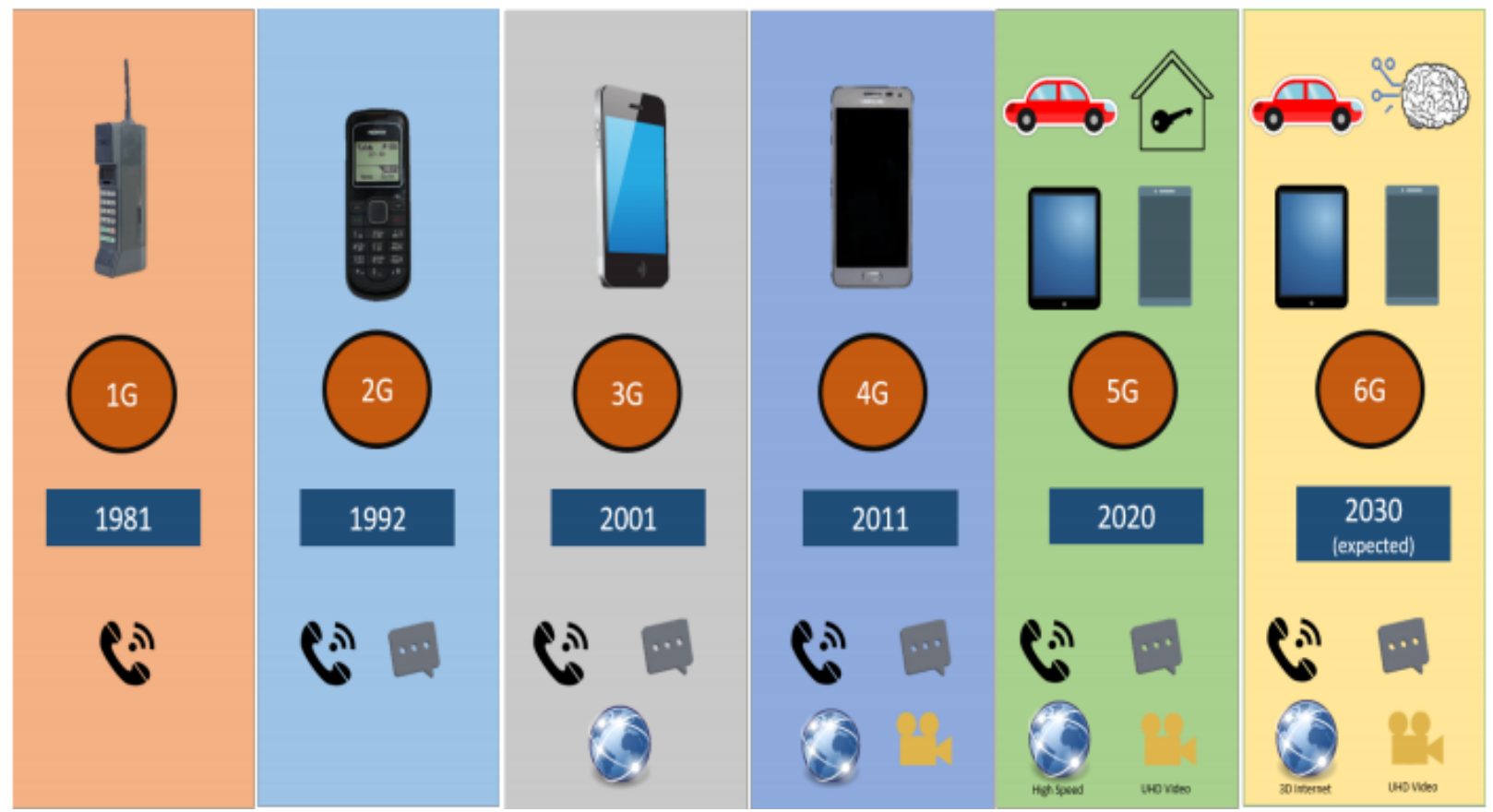

\subsubsection{G}

Figure 2.1 The evolution of mobile communication from 1G to 5G

The $1 \mathrm{G}$ versatile organizations were presented in the early 1980s and utilized simple signs for voice-just administrations. $1 \mathrm{G}$ systems utilized Frequency Division Multiple Access (FDMA) and offered information rates up to $2.4 \mathrm{kbps}$. They had helpless voice quality because of high impedance. $1 \mathrm{G}$ systems included Advanced Mobile Phone Systems (AMPS), Total Access Communication System (TACS), and Nordic Communication System (NMTS).

\subsection{2 $2 \mathrm{G}$}

The second generation (2G) mobile networks were presented in the early 1990s and were by and large thought to be computerized variants of $1 \mathrm{G}$ organizations. Alongside voice administrations, they permitted Short Message Service (SMS) and essential email administrations. These systems utilized Code Division Multiple Access (CDMA) and Time Division Multiple Access (TDMA) and offered information rates from 14.4 kbps up to 64 kbps. 2G systems included Global System for Mobile Communication (GSM) and IS-95 CDMA.

\subsubsection{G}

Third Generation (3G) is similarly considering GSM and was pushed in the year 2000. The purpose of this advancement was to offer fast data across the world. The main advancement was improved to allow data up to 14 Mbps and all the really using pack trading. It uses Wide Band Wireless Networks with which clearness is extended. It in like manner offers data organizations, admittance to TV/Video, Data, Text, and so on just as new organizations like Global Roaming is presently conceivable. It works at an extent of $2100 \mathrm{MHz}$ and has an information transmission of $15-20 \mathrm{MHz}$ used for High-speed network access and video visiting.

\subsubsection{G}

$4 \mathrm{G}$ offers a significantly better downloading pace of up to $100 \mathrm{Mbps}$. $4 \mathrm{G}$ gives a similar component preferences as $3 \mathrm{G}$ yet additionally incorporates additional organizations like Multi-Media Newspapers, sitting in front of the TV programs internet utilizing administrations, for example, Netflix with much greater lucidity because of the send/get rates of Data presently being fundamentally speedier than past ages. It acquainted us with the Mobile Social Media and Mobile App world utilized how it is today. LTE (Long Term Evolution) is viewed as a 4G development.

\subsubsection{G}

The $5 \mathrm{G}$ portable organizations are right now beginning to be actualized and intend to be multiple times quicker than current $4 \mathrm{G}$ organizations. $5 \mathrm{G}$ organizations will offer information rates up to $10 \mathrm{Gbps}$, low idleness (in milliseconds), and more noteworthy dependability. Envision that a HD film can be downloaded in only a couple seconds. This innovation can support numerous Internet of Things (IoT) empowered gadgets and keen vehicles. Productive wireless access innovation that can build throughput without expanding the data transfer capacity or densifying the cell is fundamental to accomplish the continuous requests looked by $5 \mathrm{G}$.

\subsubsection{G}

The $6 \mathrm{G}$ mobile networks are finished wireless organizations with no restriction. It is at present in the formative stage, and it will give unbelievable transmission speed in the terabit range. This innovation would require a keen reception apparatus, enormous memory in mobile phones, and colossal optical organizations. The 6G networks 
will be without cell, and it would empower man-made consciousness in wireless organizations. It isn't clear what frequency band $6 \mathrm{G}$ organizations will utilize, yet it is evident that a lot higher frequency band will be expected to expand the information rate needed for $6 \mathrm{G}$ networks.

\subsection{Requirements of the 5G Wireless Network}

The $5 \mathrm{G}$ wireless organization has not been normalized at this point. The point by point and accurate specialized particulars of this organization will be accessible sooner rather than later. Be that as it may, the accompanying specialized necessities are now acknowledged by the wireless business and the scholarly world.

\subsubsection{Coverage and Data Rate}

The 5G organization ought to keep up network whenever and anyplace with a base client experience information pace of $1 \mathrm{~Gb} / \mathrm{s}$. All in all, in light of the fact that the low-portability user equipmentchannels change substantially more gradually than those of high-versatility user equipment, they require more assets for channel state information (CSI) obtaining (i.e., decreased powerful information rate). In this way, the pinnacle information rates needed by high-and low-portability clients in the $5 \mathrm{G}$ organization can be unique. The organization should likewise guarantee a specific QoS for clients going at high rates (e.g., on high velocity trains going at $500 \mathrm{~km} / \mathrm{h}$ ), where the current organizations can't agreeably support clients. (The $4 \mathrm{G}$ organization can support versatility of up to $250 \mathrm{~km} / \mathrm{h}$.)

\subsubsection{Multiple Radio Access Technologies(RATs)}

The 5G organization won't be created to supplant current wireless organizations yet rather to progress and coordinate the current organization frameworks with new ones. In the 5G organization, the current wireless advances, including worldwide system for portable communications, third-age $(3 \mathrm{G})$, high velocity bundle access, LTE and LTE-progressed, and Wi-Fi innovations, will proceed to advance and be incorporated into a brought together system.

\subsubsection{Connected Devices}

The future $5 \mathrm{G}$ organization is required to consolidate a massive number of associated gadgets, which may reach up to multiple times that of the current wireless organization. Potential use cases in such manner are wearable figuring, machine-type communications, wireless sensors, and the Internet of Things. Critically, these associated gadgets may have various prerequisites regarding correspondence rate, postponement, and dependability.

\subsubsection{Latency}

The latency necessity is typically harder to accomplish contrasted and that of the information rate as it requests that the information be conveyed to the objective inside a given timeframe. For the $5 \mathrm{G}$ organization, the start to finish dormancy prerequisite will be on the request for $1-5 \mathrm{~ms}$.

\subsubsection{Energy and Cost Efficiency}

$5 \mathrm{G}$ wireless advancements should be intended to accomplish altogether better expense efficiency (estimated in US\$ $/ b$ ) to address portable administrators' interests about income straightening. Specifically, the energy effectiveness (estimated in $\mathrm{b} / \mathrm{J}$ ) of the $5 \mathrm{G}$ organization may should be improved by a factor of 1,000 contrasted and that accomplished by current wireless advancements.

\section{LITERATURE REVIEW}

JAVED IQBAL et al (2020):In this paper the creator clarifies about the Spectral Efficiency Techniques in Device-to-Device Communication for $5 \mathrm{G}$. Group of people yet to come organizations will oblige a lot of information traffic and lower idleness. To fulfill these needs, it is crucial for investigate current spectral utilize or present new frequency groups. Presentation of new frequency groups requires a fractional or complete difference in as of now sent framework, which will have high activity use and capital consumption. It is more helpful to discover different arrangements by focusing on gadget related arrangements. One of the answers for accomplish higher spectra productivity is through device-to-device (D2D) correspondence. This paper presents and thinks about late spectral proficiency strategies in 5G through D2D correspondence. The fundamental spotlight is on the usage of various procedures to improve range effectiveness. Besides, the difficulties in obstruction the executives, asset usage, power control, and mode choice of the proposed work are analyzed. Handing-off through gadgets has just been utilized in $4 \mathrm{G}$ to lessen blockage. Transfer base station (BS) were utilized to offload the cell traffic yet genuine capability of the transferring can be investigated by utilizing versatile based handing-off for example D2D correspondence. There are not many advancements like D2D correspondence, for example, Mobile Ad-hoc NETworks (MANET) and Cognitive Radio Networks (CRN). MANET is fundamentally an assortment of various portable hubs that make a transitory organization with no help by brought together hub. Cognitive Radio Networks is a versatile radio organization that can be arranged powerfully to identify which correspondence directs are being used and which are not and to utilize the ideal wireless channels to evade obstruction.

Yu-NgokRuyue Li et al (2020): This paper clarifies about the Beam Management in Millimeter-wave Communications for 5G. Massive MIMO is one of the promising methods to improve spectral productivity and organization execution for arriving at its focused on multi-gigabit throughput in 5G systems. For 5G New Radio 
(NR) systems, one of the key contrasts contrasted with 4G systems is the use of high frequency millimeter wave (mmWave) groups notwithstanding sub-6GHz groups. To keep the intricacy and usage cost low, half breed simple computerized bar shaping with enormous scope reception apparatus exhibit has become a typical plan way to deal with address the issue of higher engendering misfortune just as to improve spectral effectiveness in mmWave correspondence in $5 \mathrm{G}$ NR. The $5 \mathrm{G}$ NR standard is intended to adjust to various shaft framing engineering and sending situations. This paper gives the outline on pillar the board system as per the current $5 \mathrm{G}$ normalization progress. We talk about some significant difficulties of millimeter-wave communications experienced in the current 5G NR standard and present some normal improvements considered for the future past $5 \mathrm{G}$ standard.

Binqi Yang et al (2020):In this paper the creator clarifies about the 64-channel massive multiple-input multipleoutput (MIMO) handset with a completely digital beamforming (DBF) design for fifth-age millimeterwave communications is introduced in this paper. The DBF-based massive MIMO handset is worked at 28-GHz band with a 500-MHz signal transfer speed and the time division duplex mode. The recieving wire components are masterminded as a 2-D cluster, which has 16 sections (horizontal direction) and 4 lines (vertical bearing) for a superior beamforming goal in the even plane. To accomplish half-frequency component separating the flat way, another sectorial handset exhibit plan with a bowed substrate-coordinated waveguide is proposed. The deliberate outcomes show that a great RF execution is accomplished. The system execution is tried with the over-the-air strategy to check the achievability of the proposed DBF-based massive MIMO handset for high information rate millimeter-wave communications. Utilizing the bar following procedure and two surges of QAM-64 signs, the proposed millimeter-wave MIMO handset can accomplish a consistent 5.3-Gb/s throughput for a solitary client in quick portable conditions. In the multiple-client MIMO situation, by conveying 20 noncoherent information streams to eight four-channel client terminals, it accomplishes a downlink top information pace of $50.73 \mathrm{~Gb} / \mathrm{s}$ with the spectral effectiveness of $101.5 \mathrm{~b} / \mathrm{s} / \mathrm{Hz}$.

\section{PROPOSED METHODOLOGY}

With the innovation of wide scope of wireless gadgets like advanced mobile phones, tablets, and so on, which are prepared to do fast sight and sound streaming, wireless information traffic has expanded drastically. Spectral effectiveness, the normal information rate per unit data transmission turns out to be exceptionally significant. With this high pace of information traffic, blockage issues will trouble the wireless organizations sooner rather than later. Consequently, improving transfer speed productivity or spectral effectiveness gets fundamental for future wireless correspondence systems. The sort of Channel coding method, adjustment procedures and the multiple entrance strategy chose assumes significant part in improving spectral proficiency. Basically the high spectral proficiency is accomplished when higher balance request and lower code rate is chosen, prompting the need of raised execution mistake remedying code. Spectral productivity or transfer speed proficiency alludes to the measure of data that can be sent over a given transmission capacity in a particular correspondence system. The three fundamental zones where spectral proficiency can be improved is with productive channel coding strategies, data transmission effective tweak methods and massive multiple entrance procedures. The fourth exertion to improve the spectral productivity is consolidating channel coding, tweak and multiple entrance methods

\subsection{Spectral efficiency improvement technique}

Figure 4.1 portrays the model depiction for single client OFDM system which is utilized to improve the spectral effectiveness of $5 \mathrm{G}$ communication. The transmitter side of the OFDM system includes following cycles:

- Source encoding:This is needed to address the information efficiently. Different source encoding plans are there viz. Shannon-Fano, Lampel-Ziv, Hufman, and so forth. The source encoding cycle will diminish the repetition associated with information portrayals.

- Channel encoding:Channel encoding plan for example FEC is needed to identify and address the blunders at the collector end in this way making the exhibition of the system more dependable. There is an assortment of coding plans accessible which can be utilized as FEC, for example, Low-density parity check (LDPC), ReedSolomon (RS) codes, Turbo codes, convolutional codes, and so forth The FEC will add some repetition to the information, which is straightforwardly liable for the mistake decrease at the collector end. Convolutional codes are generally utilized in the OFDM actual layer as FEC codes. The encoding interaction in convolutional encoding is based on the generator framework, code rate, and imperative length. The move register is used to produce the encoded succession for a specific input arrangement by using the modulo- 2 expansion of the input grouping and substance of the move register. The disentangling interaction for the convolutional encoding utilizes the Viterbi calculation. 


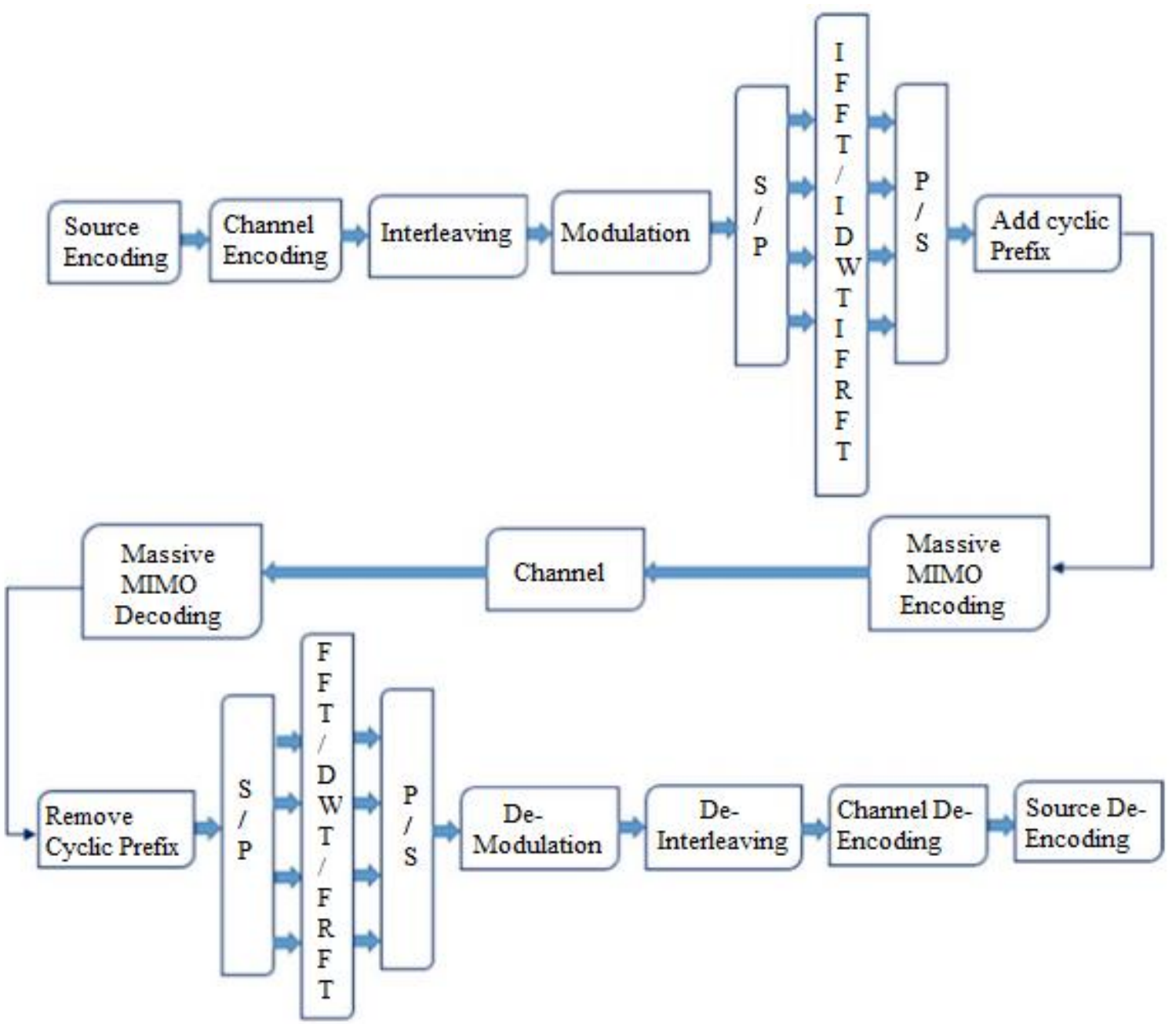

Figure 4.1 OFDM model description for improving spectral efficiency

- Interleaving:The interleaving is utilized to decrease the burst mistakes. In the OFDM actual layer, the square interleaving is utilized with block size equivalent to the encoded block size in pieces. The interleaving apportions the pieces in frequency, time, and time-frequency the two spaces to accomplish least burst blunder at the recipient end.

- Modulation:Modulation or image planning in the OFDM is achieved through the computerized adjustment plans, for example, M-ary phase shift keying (M-PSK) and M-ary quadrature amplitude modulation (MQAM). The essential utilization of planning is to plan the computerized information onto the simple transporters. In OFDM actual layer the balances which are generally utilized are BPSK, QPSK, 16-PSK, 64PSK, and QAM. The higher request tweak levels will upgrade the information rates at the expense of expanded BER.

- Serial to parallel and parallel to serial conversion: After doing the image planning the information is then isolated into ' $\mathrm{N}$ ' equal streams, where ' $\mathrm{N}$ ' is the quantity of symmetrical sub-transporters by serial to parallel conversion. After the planning of the balanced images on the symmetrical sub-transporter, the corresponding to sequential transformation is performed to ensure that the information stays in sequential structure for effective transmission over the actual channel.

- IFFT/IDWT/IFRFT:The IFFT/IDWT/IFRFT will be utilized to create the symmetrical subcarriers for the transmission of the information images. There are different sizes of IFFT referenced in the standard like 2048, $1024,512,128$, and so forth On the comparable footing, different sorts of wavelets are there in the wavelet family, for example, haar, coifet, symlet, biorthogonal, invert biorthogonal, and so forth The IFRFT is actualized by differing the estimations of rotation angle.

- Cyclic prefix:To take out the ISI the idea of the watchman span is being used in the actual layer of OFDM which is otherwise called the expansion of cyclic prefix (CP). In the cyclic prefix, every one of the images is going by the expansion of the actual image. The gatekeeper time should be picked cautiously so the multipath parts from one image won't meddle with the following image. According to the details gave by the different discussions, the gatekeeper stretch/CP for example Tg can be T/4, T/8, T/16, T/32. The decision of a specific estimation of watchman span is needy upon the measure of postpone spread in the multipath blurring channel. 
At the collector, all the cycles done at the transmitter end will be turned around beginning from disposing of the watchman stretch. At that point the images will be handled by FFT/DWT/IFRFT followed by channel assessment/balance. At that point the pieces will be recuperated back from the images by using the image deplanning (digital demodulation) plans. After this, the pieces will be modified by the square interleaver followed by the FEC deciphering to moderate the excess present in the information. In the last, the de-randomizer will descramble the information to introduce the information in the first structure to the upper layer of the order.

\subsection{Massive MIMO}

MIMO systems are an indispensable piece of current wireless systems, and lately they have been utilized broadly to accomplish high spectral effectiveness and energy productivity. Prior to the presentation of MIMO, singleinput-single-output systems were for the most part utilized, which had exceptionally low throughput and couldn't support countless clients with high dependability. To oblige this massive client interest, different new MIMO innovation like single-client MIMO (SU-MIMO), multi-client MIMO (MU-MIMO) and organization MIMO were created. Be that as it may, these new innovations are additionally insufficient to oblige the always expanding requests. The wireless clients have expanded dramatically over the most recent couple of years, and these clients create trillions of information that should be taken care of productively with greater dependability.

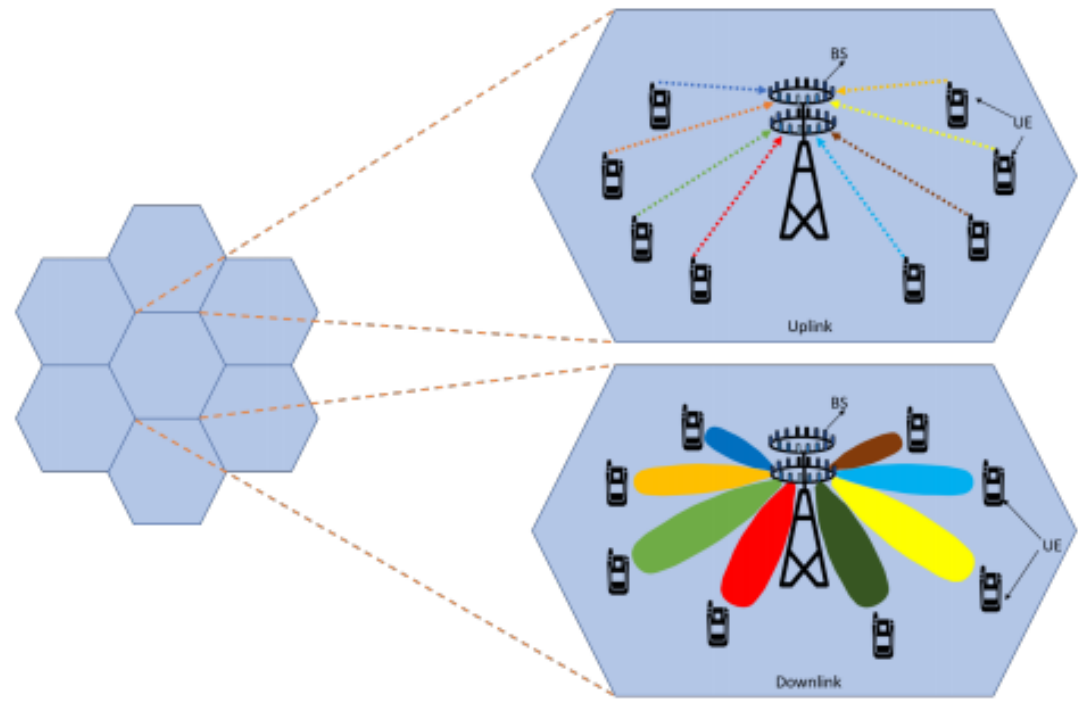

Figure 4.2. Massive MIMO uplink and downlink

Massive MIMO is the most dazzling innovation for $5 \mathrm{G}$ and past the wireless access period. Massive MIMO is the headway of contemporary MIMO systems utilized in current wireless organizations, which groups together hundreds and even large number of antennas at the base station and serves many clients at the same time. The additional antennas that massive MIMO uses will help center energy into a more modest area of room to give better spectral proficiency and throughput. Massive MIMO downlink and the uplink system is appeared in Figure 4.2. As the quantity of radio wire increments in a massive MIMO system, emanated radiates become smaller and spatially engaged toward the client. These spatially engaged reception apparatus radiates increment the throughput for the ideal client and lessen the obstruction to the neighboring user.

\subsubsection{Uplink Transmission}

The uplink channel is utilized to send information and the pilot signal from the client terminal to the base station as demonstrated in Figure 4.3(a). Allow us to consider a massive MIMO uplink system outfitted with M antennas at the base station and at the same time speaking with $\mathrm{N}(\mathrm{M}>\mathrm{N})$ single-reception apparatus clients. On the off chance that the sign sent by the client or the deterministic pilot sign to assess the channel is $\mathrm{x} \in \mathrm{CN}$, the sign got at the base station during uplink is given as:

$$
y=H x+n_{u p l i n k}
$$

where $\mathrm{y} \in \mathrm{CM}$ is the sign gotten at the base station, $\mathrm{H}$ is the channel vector between the client terminal and the base station, and components of $\mathrm{H} \in \mathrm{C}^{\mathrm{M} \times \mathrm{N}}$ are autonomous and indistinguishably dispersed with zero mean and unit fluctuation, that is, $\mathrm{H} \sim \mathrm{CN}(0,1)$. The obstruction added is autonomous of the client signal $\mathrm{x}$, however it tends to be subject to the channel $\mathrm{H}$. The impedance added is autonomous of the client signal $\mathrm{x}$, however it tends to be subject to the channel $\mathrm{H}$.

$$
n_{\text {uplink }}=n_{\text {uplink-interference }}+n_{\text {noise }} \text {. }
$$




\subsubsection{Downlink Transmission}

The downlink channel is utilized to communicate information or gauge the channel among client and base station. The base station utilizes preparing pilots to gauge the channel. A downlink transmission with a few user equipment and a base station is appeared in Figure 4.3(b). Allow us to consider a downlink massive MIMO system, where base station furnished with $\mathrm{M}$ antennas, and it is serving $\mathrm{N}$ clients having a solitary antenna at the same time. The base station sends free data to multiple clients at the same time. The sign got, $y k \in \mathrm{C}^{\mathrm{M} \times 1}$ at the kth client is:

$$
y_{k}=h_{k} x_{k}+n_{\text {downlink }}
$$

wherehk is a channel vector between kth client and base station, whose components are autonomous and indistinguishably circulated with zero mean and unit fluctuation, that is, $\mathrm{h} \sim \mathrm{CN}(0,1)$. $\mathrm{xk} \in \mathrm{CM}$ is the sign communicated by base station for client $\mathrm{k}$ and, $\mathrm{n}_{\text {downlink }}$ is the extra clamor which is made out of the recipient commotion $\mathrm{n}_{\text {noise }} \sim \mathrm{CN}(0, \sigma 2 \mathrm{I})$ and the impedance during downlink $\mathrm{n}_{\text {downlink-interference }}$ brought about by sending all the while to different clients and is given as:

$$
n_{\text {downlink }}=n_{\text {downlink-interference }}+n_{\text {noise }}
$$

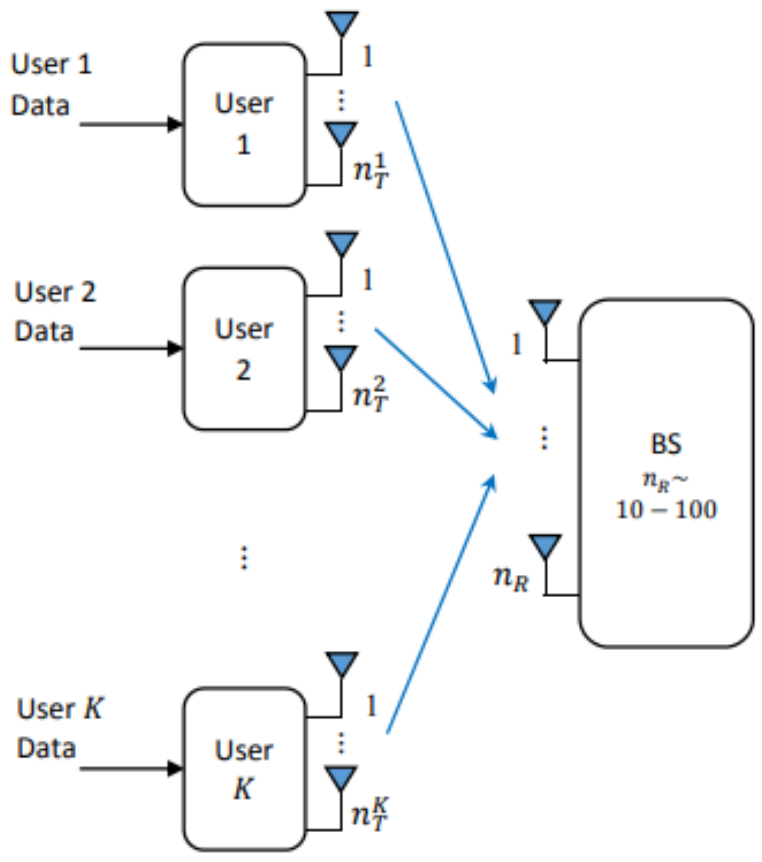

(a)

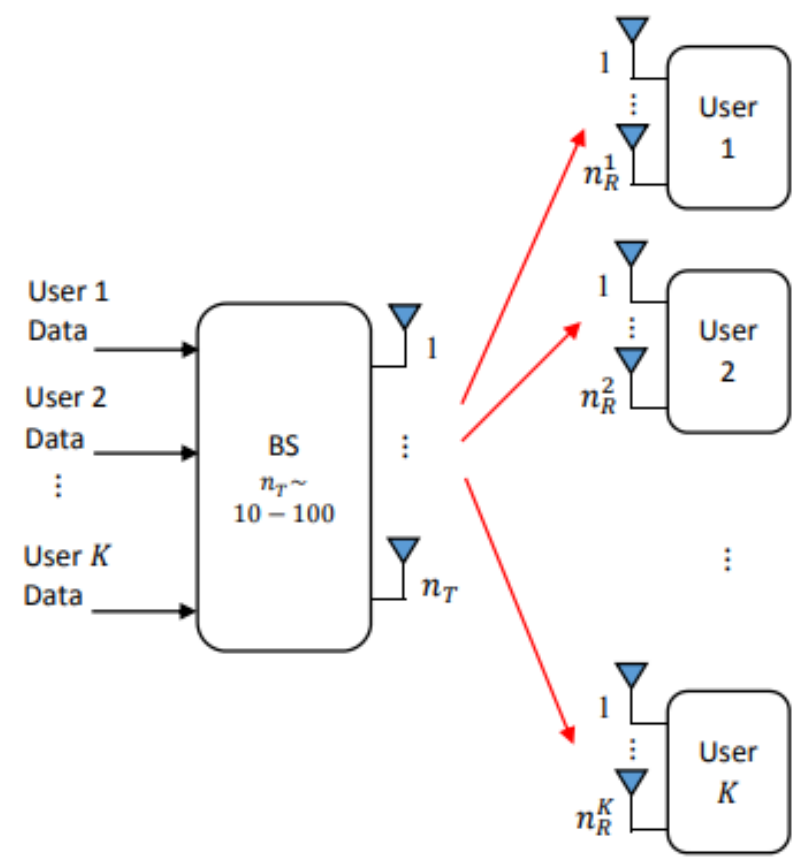

(b)

Figure 4.3. Massive MIMO uplink and downlink operation. (a) Uplink (b) Downlink.

\subsection{Increase in system capacity, spectral efficiency and energy efficiency}

Massive MIMO wireless system alludes to an enormous number of antennas with significant degrees, e.g., at least 100 are prepared at the cell BSs to upgrade the system limit, spectral and energy effectiveness in both the downlink and uplink communications. Fundamentally, massive MIMO BSs abuse an exceptionally serious level of spatial multiplexing to improve the system limit. As the BSs are sent with countless reception apparatus clusters, straightforward direct beamforming/precoding is conceivable to expand the spectral and energy proficiency. A few reenactment works have been accounted for to dissect the impact of huge number of radio wire clusters on spectral proficiency. A uniform linear array (ULA) with 400 antenna wire components serving 10 client terminals was recreated at $2.6 \mathrm{GHz}$ by utilizing two diverse precoding plans, eigenbeamforming (BF) and zero forcing (ZF). Spectral proficiency of $58 \mathrm{~b} / \mathrm{s} / \mathrm{Hz}$ and $48 \mathrm{~b} / \mathrm{s} / \mathrm{Hz}$ has been accomplished for ZF and $\mathrm{BF}$ respectively. The spectral productivity of exceptionally huge MU-MIMO that comprises of $500 \mathrm{BS}$ antennas serving 10 clients all the while based on LTE standard. Basic straight precodings have been embraced in the smiulations, for example, maximum ratio combining (MRC), minimum mean square error (MMSE) and ZF that yield spectral proficiency of $42 \mathrm{~b} / \mathrm{s} / \mathrm{Hz}$ (amazing channel state data, CSI) and 28b/s/Hz (flawed CSI). In the interim, 2D rectangular reception apparatus cluster design with antennas expanding up to 250 was recreated to serve 10 clients under Wi-Fi and LTE groups.

Spectral productivity of $130 \mathrm{~b} / \mathrm{s} / \mathrm{Hz}$ (LTE) and $110 \mathrm{~b} / \mathrm{s} / \mathrm{Hz}$ (Wi-Fi) with ZF precoding, while 22b/s/Hz (LTE) and 19b/s/Hz (Wi-Fi) with MRC precoding have been recorded. Other than that, there have been some new viable 
exhibitions on check of the massive MIMO advantage to understand its hypothetical interest, for example the proving grounds. The 64-components Argos system fit for serving 15 clients at the same time has accomplished a limit up to 85 pieces/s/Hz. The RUSK Lund proving grounds were worked at Lund University comprises of a round and hollow cluster and straight exhibit. Every reception apparatus cluster is furnished with 128 antennas components speaking with 4 clients at $2.6 \mathrm{GHz}$ by actualizing MRT precoding/beamforming plan. Beginning outcomes showed that the spectral productivity for direct cluster is $48 \mathrm{~b} / \mathrm{s} / \mathrm{Hz}$ and for tube shaped exhibit is 32b/s/Hz. Unmistakably many-antennas BS can improve the spectral limit contrasted with utilizing a solitary radio wire. The spectral limit increments almost straight with the quantity of BS antennas serving multiple clients at the same time. Other than that, multiple precoding methods have been utilized in the reenactments or analyses to exhibit the increment in spectral limit. Generally, ZF precoding can proceed just as BF or MRC precoding with a huge number.

\section{RESULTS}

In single m-MIMO just as organization MIMO with full collaboration all clients are served all the while. For the circulated MIMO without collaboration the user equipment(UE) associates with the exhibit with the most extreme normal SNR and precoding is performed locally by that particular cluster while treating impedance of other downlink transmissions as commotion. In the event that more user equipmentassociate with one exhibit as there are communicate antennas, the client hardware are planned with channel decay utilizing limit upperbound. As found in Figure 5.1 single m-MIMO (solid lines) brings about the most minimal Spectral efficiency (SE), dispersed MIMO without collaboration (dashed lines) gives critical SE gains over the single m-MIMO case, though network MIMO with full participation (dotted lines) yield the most noteworthy SE. The completely stacked situation $\left(\mathrm{N}_{\mathrm{T}}=\mathrm{N}_{\mathrm{UE}}=20\right)$ uncovers the most minimal SE contrasted with setups with higher number of antennas. On the off chance that NT is simply expanded somewhat by 4 to 24 TX antennas the SE is multiplied, subsequently for this organization situation a $20 \%$ increment in TX antennas above baseline arrangement $\left(\mathrm{N}_{\mathrm{T}}=\right.$ $\mathrm{N}_{\mathrm{UE}}$ ) brings about $100 \%$ limit gain.

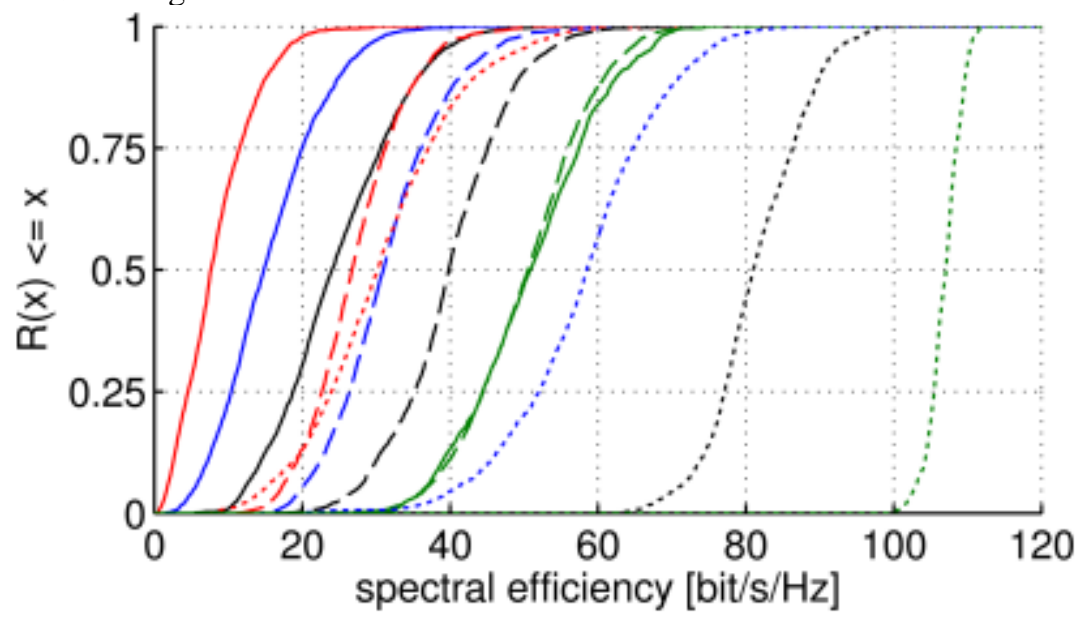

Figure 5.1 Spectral Efficiency for 20 UEs (red: $N_{T}=20$, blue: $N_{T}=24$, black: $N_{T}=36$ and green: $N_{T}=200$ total transmit antennas; solid: single m-MIMO, dashed: distributed m-MIMO, dotted: network mMIMO)

Adding more antennas expands the presentation however with diminishing increase per each extra reception apparatus, for example a 1000\% increment in TX antennas brings about under 1000\% limit acquire. In Figure 5.1 conveyed MIMO with 24 TX antennas brings about a comparative execution as organization MIMO with just 20 TX antennas. Subsequently potential equipment reserve funds because of less antennas must be offset with extra expense needed for backhaul. Moreover Figure 5.1 uncovers that for 20 TX antennas the contrast between single $\mathrm{m}-\mathrm{MIMO}$ and circulated MIMO is triple while for $200 \mathrm{TX}$ antennas the distinction between single m-MIMO and dispersed m-MIMO is irrelevant. For a proportion of 10 of communicate antennas to UEs the SE cdf bend is moving toward a stage work, for example cell edge clients (5\%tile) and 95\%tile clients notice practically a similar rate.

\section{CONCLUSION}

The wireless local area is a couple of steps from the execution of $5 \mathrm{G}$ wireless organizations, which are relied upon to give universal correspondence capacities. The massive development in rush hour gridlock requests for portable information administrations has made Massive MIMO is viewed as one of the critical advances in fifth generation (5G) supplanting the regular MIMO system. Massive MIMO depends on the law of huge number of antennas that can immensely improve the sign strength, increment information rates and improve signal unwavering quality. Since the proposed technique shows better execution for higher number of antennas, this 
system can be adaptively utilized for Massive MIMO 5G systems whose fundamental component is huge number of communicating antennas. The expansion in spectral effectiveness will improve the throughput of the system which straightforwardly serves the Enhanced Mobile Broad band use instance of the 5G assistance. In the massive MIMO, it is vital for straight discovery to play out the zero-compelling to permit the solid location to collect excellent execution through empowering an increment in the component of exhibits which moderate the preparing issue and considered as an okay territory. Massive MIMO economically expanded the spectral efficiency and enhance capacity and coverage.

\section{REFERENCES}

1. JAVED IQBAL, MUHAMMAD A. IQBAL, AWAIS AHMAD and MURAD KHAN (2020), "Comparison of Spectral Efficiency Techniques in Device-to-Device Communication for 5G". IEEE Commun. Mag., vol. 52, no. 2, pp. 74-80, Feb. 2020.

2. Li, Y.-N. R., Gao, B., Zhang, X., \& Huang, K. (2020). Beam Management in Millimeter-wave Communications for 5G and beyond. IEEE Access, 1-1. doi:10.1109/access.2020.2963514

3. Yang, B., Yu, Z., Lan, J., Zhang, R., Zhou, J., \& Hong, W. (2020). Digital Beamforming-Based Massive MIMO Transceiver for 5G Millimeter-Wave Communications. IEEE Transactions on Microwave Theory and Techniques, 66(7), 3403-3418. doi:10.1109/tmtt.2020.2829702

4. Y. Yang et al., "Fast Optimal Antenna Placement for Distributed MIMO Radar with Surveillance Performance," vol. 22, no. 11, pp. 1955-1959, 2019.

5. H. Ji et al., "Overview of full-dimension MIMO in LTE advanced pro," IEEE Commun. Mag., vol. 55, no. 2, pp. 176-184, Feb. 2020.

6. R. Fan, Y. J. Yu, and Y. L. Guan, "Orthogonal frequency division multiplexing with generalized index modulation," in IEEE Global Commun. Conf., Austin, TX, USA, Dec. 2019, pp. 3880-3885.

7. S. Zhang, P. Y. Kam, J. Chen, and C. Yu, Bit-error rate performance of coherent optical M-ary PSK/QAM using decisionaided maximum likelihood phase estimation, Department of Electrical \& Computer Engineering, National University of Singapore,117576, Singapore. 24 May 2020 / Vol. 18, No. 12 / OPTICS EXPRESS 12088

8. E. Basar, "Index modulation techniques for 5G wireless networks,"IEEECommun. Mag., vol. 54, no. 7, pp. 168-175, June 2019.

9. VENGATESAN, K., KUMAR, E., YUVARAJ, S., TANESH, P., \& KUMAR, A. (2020). An Approach for Remove Missing Values in Numerical and Categorical Values Using Two Way Table Marginal Joint ProbabilityInternational Journal of Advanced Science and Technology, 29(5), 2745-2756

10. Qiao, X. Shen, J. Mark, Q. Shen, Y. He, and L. Lei, “Enabling device to device communications in millimeter-wave 5G cellular networks," IEEE Commun. Mag., vol. 53, no. 1, pp. 209_215, Jan. 2020

11. K. Doppler, M. Rinne, C. Wijting, C. B. Ribeiro, and K. Hugl, “Device-todevice communication as an underlay to LTE-advanced networks,' IEEE Commun. Mag., vol. 47, no. 12, pp. 42-49, Dec. 2020.

12. Mallikalava, V., Yuvaraj, S., Vengatesan, K., Kumar, A., Punjabi, S., \& Samee, S. (2020). Theft Vehicle Detection Using Image Processing integrated Digital Signature Based ECU. In 2020 Third International Conference on Smart Systems and Inventive Technology (ICSSIT) (pp. 913-918). 